\title{
Experiencias
}

\section{La pandemia: oportunidad de aprendizaje. Estrategias llevadas a cabo por el Equipo Específico de Atención al Alumnado con Discapacidad Visual de Málaga}

The pandemic as a learning opportunity: strategies implemented by the Team for the Specific Support of Students with Visual Disability, Málaga

\section{M. Montiel Campos, E. Páez Romero, A. Vioque Fernández, M. C. Campanario Pérez, M. Urbano Labajos}

\section{Resumen}

Como profesionales de la educación, queremos mostrar el lado positivo de las cosas y, de este modo, enseñar a nuestro alumnado con discapacidad visual a encontrar oportunidades, estrategias para superar la dificultad, con el fin de pensar en un futuro mejor, «su futuro». Durante esta pandemia, provocada por el coronavirus SARS-CoV-2, hemos sido testigos de que, frente a la adversidad, el ser humano es capaz de buscar y encontrar nuevas formas de comunicación, de aprendizaje, de relaciones sociales, etc. Desde el Equipo Específico de Atención al Alumnado con Discapacidad Visual de Málaga hemos dado sobrada cuenta de ello convirtiendo esta crisis en una oportunidad de aprendizaje. Hemos sido capaces de adaptar nuestra forma de enseñanza presencial a nuevas metodologías a distancia, establecer nuevas formas de comunicación con las familias, crear y compartir recursos. Así como lanzar un blog educativo y crear perfiles en redes sociales estableciendo lazos de comunicación entre toda la comunidad educativa con el fin de conseguir una educación inclusiva.

\section{Palabras clave}

Herramientas digitales. Pandemia. Redes educativas. Blog educativo. Redes sociales. 


\begin{abstract}
As educational professionals, the authors tend to view the positive side of events to teach their students with visual disability how to seek opportunities, design strategies to surmount obstacles and aim for a better future, 'their future'. The SARS-CoV-2-induced pandemic has attested to humanity's capacity, when confronted with adversity, to seek and find new forms of communicating, learning and interacting socially. The Team for Specific Support for Students with Visual Disability in Málaga stands as ample proof of that capacity, having turned the crisis into a learning opportunity. The team has proven able to adapt its in-person teaching model to new remote methodologies, establish new vehicles for communicating with families and create and share resources. They have also launched an educational blog, created profiles in social networks and established communication channels with the entire educational community in pursuit of inclusive education.
\end{abstract}

\title{
Key words
}

Digital tools. Pandemic. Educational networking. Educational blog. Social networks.

\section{Introducción}

13 de marzo de 2020: momento en el que se declara el estado de alarma en España debido a la crisis sanitaria causada por el coronavirus SARS-CoV-2. Se cierra toda actividad no esencial, se establecen medidas de confinamiento domiciliario y los centros educativos cierran sus puertas, en principio, durante dos semanas, para intentar contener la transmisión del virus, ya en esos momentos declarada como pandemia por la Organización Mundial de la Salud.

La covid-19 ha supuesto una prueba de estrés para todos, y un golpe duro a todos los niveles socioeconómicos. En el sector público, no solo el sistema sanitario se ha visto comprometido, también el sistema educativo ha tenido que asumir el nuevo reto de la educación a distancia, lo que ha significado un desafío para el profesorado y, especialmente, para los profesionales del Equipo Educativo de Atención al Alumnado con Discapacidad Visual (EEAADV) de Málaga, al encontrarnos ante el reto de atender a nuestro alumnado ciego o con baja visión de forma no presencial; un alumnado heterogéneo en edad, etapa educativa, nivel curricular, manejo de las tecnologías, diferentes capacidades, herramientas específicas de cada alumno para el acceso al currículo, etc.

Montiel, M.M., Páez, E., Vioque, A., Campanario, M.C., y Urbano, M. (2021). La pandemia: oportunidad de aprendizaje; estrategias llevadas a cabo por el Equipo Específico de Atención al Alumnado con Discapacidad Visual de Málaga. RED Visual: Revista Especializada en Discapacidad Visual, 77, 284-293. https://doi.org/10.53094/BLYU8792. 
En un primer momento, desde el EEAADV se abordó esta nueva situación haciendo uso de las herramientas tecnológicas y tiflotecnológicas con las que se contaba; sin embargo, fuimos conscientes de que la situación precisaba de estrategias adaptadas a los nuevos modos de enseñanza a distancia que se nos planteaban.

Fueron momentos convulsos, llenos de dudas e inquietudes, pues una gran parte de nuestra intervención consiste en la atención directa con el alumnado. Nos preguntamos cómo íbamos a atender sus necesidades en la distancia, cómo se iba a ofrecer en cada uno de los centros educativos la formación online y cómo adaptaríamos nuestra formación-intervención a la situación sobrevenida sin afectar al asesoramiento, intervenciones y/o adaptaciones que, desde el equipo, veníamos realizando desde hace más de 25 años y, así, seguir contribuyendo en el proceso de enseñanza-aprendizaje en la nueva modalidad online.

Las primeras comunicaciones con los equipos docentes y con las familias iban encaminadas a transmitir calma, procurar que se sintieran apoyados, así como acompañar, escuchar, establecer lazos emocionales. Todos estábamos viviendo una situación muy dramática, pero, al mismo tiempo, como especialistas, teníamos que dar respuesta a todas las demandas que iban surgiendo, como crear, adaptar y/o organizar materiales específicos que luego el centro entregaría a las familias con objeto de que nuestro alumnado pudiera seguir el currículo en casa con los recursos necesarios, establecer las vías de comunicación y los distintos perfiles de plataformas que íbamos a utilizar...

Por suerte, la capacidad de reinvención es una de las habilidades innatas que tenemos los docentes: sabemos trabajar para encontrar soluciones y crear nuevos entornos de aprendizaje para nuestro alumnado. Así pues, en cuestión de días, nos adaptamos a las diversas plataformas de formación a distancia (Google Classroom, Moodle, Zoom, Meet, etc.) para salvar la nueva situación.

Gracias a estas plataformas se trabajó codo con codo con los equipos docentes, equipos directivos, familias y alumnado, con el objetivo principal de conseguir la igualdad de oportunidades en esta nueva formación telemática que acababa de irrumpir como medio de enseñanza-aprendizaje. Usamos numerosos recursos, plataformas, estrategias a distancia personalizadas a las necesidades de nuestro alumnado instaladas en nuestros ordenadores a demanda de la atención que requería cada caso en particular. De la noche a la mañana, no sin falta de motivación y vocación, fuimos capaces de asumir el reto y adaptarnos a la enseñanza telemática dirigida a personas

Montiel, M.M., Páez, E., Vioque, A., Campanario, M.C., y Urbano, M. (2021). La pandemia: oportunidad de aprendizaje; estrategias llevadas a cabo por el Equipo Específico de Atención al Alumnado con Discapacidad Visual de Málaga. RED Visual: Revista Especializada en Discapacidad Visual, 77, 284-293. https://doi.org/10.53094/BLYU8792. 
ciegas o con baja visión. Pero, como dijo el profesor Howard G. Hendricks, «La enseñanza que deja huella no es la que se hace de cabeza a cabeza, sino de corazón a corazón».

En este curso 2020-2021 se siguen sufriendo las consecuencias de esta pandemia. Sin embargo, ahora estamos mejor entrenadas, más familiarizadas con todas las herramientas digitales que tenemos a nuestra disposición, sabemos que estamos más cerca de recuperar todo aquello que la covid nos ha obligado a modificar, y hemos aprendido cuán importante es cuidarnos y trabajar unidas en pro de un objetivo común. En este sentido, estamos más capacitadas para vislumbrar las cosas positivas que hemos experimentado y las cosas buenas que hemos aprendido en estos tiempos de confinamiento y pandemia. Algunas de ellas han sido:

- Las familias están más involucradas en el proceso de enseñanza-aprendizaje de sus hijos (ayuda a la realización de tareas, conciencia del grado y ritmo de trabajo en el aula, etc.) y tienen una mejor o mayor valoración de la labor docente.

- Los centros educativos son más accesibles, en algunos sentidos, para nuestro alumnado: menor contaminación acústica en las aulas - que favorece la atmósfera de trabajo-, señalización de los espacios y gestión de la movilidad, puesta en valor de las tecnologías de la comunicación en el ámbito escolar o educativo...

- Los niveles de contaminación medioambiental han bajado.

- La mejora en la higiene personal, la limpieza de espacios públicos, el uso de la mascarilla y la distancia social han rebajado la incidencia de enfermedades como la gripe y los resfriados.

- Hemos ampliado nuestros conocimientos, no solo en informática, sino en biología, medicina, psicología, etc.

- Cuidamos nuestra alimentación y nuestra salud como no lo hacíamos antes.

- Se ha sido testigo de la gran creatividad y solidaridad que nos invade.

- Tenemos más tiempo para nosotros y para compartir en familia, nos comunicamos y nos conocemos mejor.

Montiel, M.M., Páez, E., Vioque, A., Campanario, M.C., y Urbano, M. (2021). La pandemia: oportunidad de aprendizaje; estrategias llevadas a cabo por el Equipo Específico de Atención al Alumnado con Discapacidad Visual de Málaga. RED Visual: Revista Especializada en Discapacidad Visual, 77, 284-293. https://doi.org/10.53094/BLYU8792. 


\section{Actuaciones desarrolladas durante la pandemia}

En palabras de Einstein, «Es en la crisis donde nace la inventiva, los descubrimientos y las grandes estrategias» y «Quien supera la crisis se supera a sí mismo sin quedar superado».

La situación actual ha reforzado nuestra creencia de que familia y educación tienen que ir de la mano, caminar en la misma dirección, trabajar de manera coordinada. A día de hoy, se puede confirmar que el aprendizaje puede salir de la escuela y que puede darse en otros escenarios a través de plataformas virtuales con la ayuda de las familias.

El primer mensaje que les mandamos estaba cargado de tranquilidad y ánimos:

Buenas tardes familia: Atendiendo a las medidas dictadas por las autoridades con el fin de asegurar nuestra salud y poner freno a la pandemia de la covid-19, y habiéndose ampliado el estado de alerta sanitaria, quedando suspendidas las actividades escolares, quedo a vuestra disposición para cualquier duda y/o consulta a través de mi teléfono personal y/o correo electrónico. Un saludo y mucha salud. Espero que os encontréis todos bien. Un abrazo. Firmado: Maestra del Equipo Específico de Discapacidad Visual de Málaga.

Sin embargo, la situación de confinamiento prevista para unas semanas se convirtió en meses de confinamiento domiciliario, en el que se siguieron intercambiando mensajes con las familias, reforzando el binomio escuela-familia. Rescatamos otro de tantos mensajes que enviamos durante esos días:

Buenas tardes familia: Tras haber pasado este confinamiento, nos queda la recta final para recuperar nuestra añorada normalidad. Os animo a seguir trabajando desde casa, estableciendo una rutina que os permita realizar algunas tareas escolares, hacer un poco de ejercicio físico y disfrutar de la compañía de vuestros familiares. Son muchas y diversas las plataformas de enseñanza online, programas de televisión, aplicaciones de videollamada y material curricular con el fin de haceros más llevadero el día a día y ayudaros. Aunque reconocemos que es una situación muy complicada y que no todos tenemos todos los recursos y posibilidades a nivel tecnológico. Igualmente, informaros de que estamos en contacto con los tutores y el equipo educativo de vuestros hijos. Es mucho el trabajo y el esfuerzo que habéis realizado hasta la fecha, y, por ello, debemos dar el empujón final. iÁnimo, y ya queda menos! Todo saldrá bien. Firmado: Maestra del Equipo Específico.

Sin lugar a dudas, otra de las grandes protagonistas de esta pandemia causada por la covid-19 han sido las tecnologías de la información y la comunicación. En nuestro caso, no la suerte, sino la adecuada, acertada y conveniente formación que en este campo recibe nuestro alumnado, comenzando su enseñanza desde muy temprana

Montiel, M.M., Páez, E., Vioque, A., Campanario, M.C., y Urbano, M. (2021). La pandemia: oportunidad de aprendizaje; estrategias llevadas a cabo por el Equipo Específico de Atención al Alumnado con Discapacidad Visual de Málaga. RED Visual: Revista Especializada en Discapacidad Visual, 77, 284-293. https://doi.org/10.53094/BLYU8792. 
edad, permite alcanzar la competencia digital tan necesaria a la hora de acceder al currículo escolar. De hecho, durante esta pandemia, y con las nuevas formas de educación, se ha podido evaluar y constatar cómo años de trabajo incansable por parte de alumnado, familias, especialistas en tiflotecnología y equipos docentes, están dando su fruto, y han conseguido que nuestro alumnado esté, en muchos casos, «mejor» preparado en lo relacionado con las herramientas digitales de acceso al currículo que el resto de compañeros que no tuvieron ni encontraron la necesidad de hacer uso de las mismas hasta que nos sorprendió la formación a distancia. Sin duda, este punto ha sido y es fundamental para que el alumnado con discapacidad visual pueda seguir las clases online y, de esta forma, convertirse en estudiantes más autónomos. Por otro lado, el profesorado ha tomado conciencia de la necesidad de usar el ordenador - particularmente en el caso del alumnado que desde el equipo atendemos- y de la necesidad de adaptar la metodología de enseñanza: en la medida en que existe alumnado con discapacidad visual, o cualquier otro tipo de diversidad funcional, el aula cambia y/o la situación socioeducativa cambia por completo, como nos ha ocurrido actualmente, para garantizar el acceso a la educación.

Asimismo, en todos estos meses de confinamiento, se compartieron materiales con familias, equipos, profesionales y se vio la necesidad de hacer un banco de recursos con todo lo que se estaba compartiendo: mensajes con materiales elaborados para nuestro alumnado, actividades que llegaban a través de diversas plataformas, informaciones que teníamos que ofrecer a las familias... Los móviles y equipos informáticos personales se estaban convirtiendo en un cajón de sastre con miles de mensajes diarios desordenados. El WhatsApp era un batiburrillo de enlaces a actividades, fotos y documentos que, de alguna manera, se tenían que ordenar, pues eran muy interesantes y queríamos conservarlos, clasificarlos y organizarlos para poder recurrir a esa información. Esta necesidad de poner orden y de recopilar esa información que, generosamente, se compartía entre el equipo educativo nos hizo pensar en la posibilidad de crear un blog donde quedarán recogidos todos los documentos y aportaciones que iban llegando y, de este modo, utilizar esta herramienta como medio de comunicación con la comunidad educativa.

Los blogs o bitácoras permiten, de manera fácil y rápida, que cualquier persona publique en un espacio propio sus ideas y las comparta con otras personas en la red. El formato blog se ha generalizado como el más aceptado medio de publicación, espacio personal, profesional o grupal, y se ha dejado notar en ámbitos como el educativo. 
La Consejería de Educación de la Junta de Andalucía, a través del servicio gratuito de blogs Averroes (https://blogsaverroes.juntadeandalucia.es), pone a disposición de toda la comunidad educativa (docentes, centros educativos, ampas) un espacio propio para la creación, administración y publicación de blogs y sitios web utilizando WordPress, de manera que permite difundir experiencias educativas, publicar contenidos y actividades educativas o utilizarlo como recurso didáctico en el aula. Aprovechando este recurso, se solicitó un espacio para la creación de nuestro blog, se creó el perfil en redes del EEAADV de Málaga y, en mayo de 2020, nació el blog del equipo de Málaga, cuya dirección es https://blogsaverroes.juntadeandalucia.es/equipoespecificodiscapacidadvisualmalaga/ (ver Figura 1).

Figura 1. Captura de pantalla de la página principal del blog

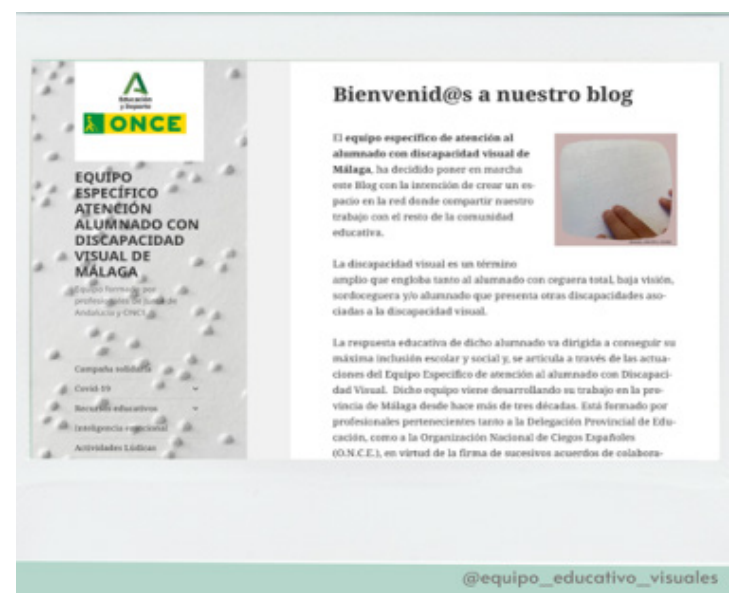

Comenzaba así nuestra andadura en este mundo virtual, en el que íbamos aprendiendo mientras se publicaban nuestras primeras entradas en el blog: «El Equipo Específico de Atención al Alumnado con Discapacidad Visual de Málaga ha decidido poner en marcha este blog con la intención de crear un espacio en la red donde compartir nuestro trabajo con el resto de la comunidad educativa». Con este mensaje comenzábamos a familiarizarnos con un entorno digital que nos ofrecía la oportunidad de seguir en contacto con la comunidad educativa y establecer vías de comunicación entre los profesionales que formamos parte del equipo específico.

Una de las primeras publicaciones en el blog daba cobertura a una iniciativa solidaria, a la que desde el equipo de Málaga quisimos unirnos y darle difusión a través de esta red social. Se trataba del proyecto Boquitas bien tapadas y sonrisa en la mirada, llevado a cabo por una compañera del equipo. De este modo, se hizo llegar mascarillas 
por correo a nuestros alumnos, junto a un mensaje de ánimo y un enlace a la canción Resistiré a través de un código accesible QR.

Se sucedieron más publicaciones, en las que se abordaron temáticas muy diversas. Entre ellas, se pueden destacar publicaciones en las que se compartió cómo construir una caja de luz o una linterna mágica, actividades para hacer en familia durante el confinamiento, se difundió una entrada en la que nuestra alumna Ana cumple su sueño de cantar con David Bisbal y Aitana, se realizó una entrevista al ganador del concurso Prometeo, se habló sobre acromatopsia, sobre sordoceguera, se dio a conocer la tecnología de NaviLens... Actualmente, ya son más de 36 publicaciones las que se pueden visitar en el blog, todas ellas teniendo en cuenta la accesibilidad.

A través de estas publicaciones se quiso dar visibilidad a diversos aspectos de la discapacidad visual desde un punto de vista educativo, ya que el equipo está formado por profesionales con distintos intereses, motivaciones y puntos de vista. Esta diversidad le confiere una riqueza que es, sin duda, una de las claves para ofrecer y plasmar en el blog una gran variedad de temas.

Paralelamente a la creación del blog se crearon los perfiles del equipo específico en la red social Instagram, @equipo_educativo_visuales, y en Facebook, Eeaadv_malaga (ver Figura 2). Comenzamos a realizar nuestras primeras publicaciones a través de nuestro perfil, abriendo una ventana para mostrar el trabajo que llevamos a cabo con nuestro alumnado, los materiales que utilizamos o las distintas metodologías, entre otras.

Figura 2. Captura de pantalla de los perfiles de redes sociales

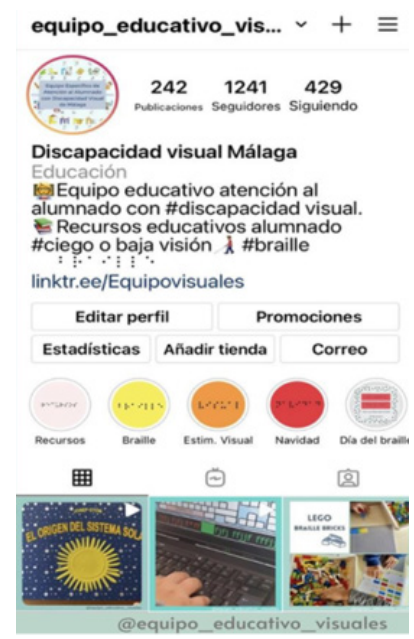

Montiel, M.M., Páez, E., Vioque, A., Campanario, M.C., y Urbano, M. (2021). La pandemia: oportunidad de aprendizaje; estrategias llevadas a cabo por el Equipo Específico de Atención al Alumnado con Discapacidad Visual de Málaga. RED Visual: Revista Especializada en Discapacidad Visual, 77, 284-293. https://doi.org/10.53094/BLYU8792. 
Publicación tras publicación, tanto la página de Instagram como la de Facebook crecen en número de seguidores $y$, al mismo tiempo, tomamos conciencia de la necesidad de estos perfiles - a los que pueden recurrir tanto profesionales y familias como alumnado ante cualquier demanda que puedan tener-, de poder compartir nuestras experiencias con el alumnado con discapacidad visual y de dar visibilidad tanto a nuestro trabajo como al colectivo de personas ciegas o con baja visión.

\section{Conclusiones}

La creación del blog y las redes sociales es un proyecto innovador y novedoso que no solo ha tenido gran aceptación en la red sino también entre los profesionales del equipo. Tanto es así que, en este curso 2020-2021, se convierte en un plan educativo, formando parte de nuestro proyecto educativo anual, con los objetivos de compartir la información con profesionales tanto dentro como fuera del equipo, ser un banco de recursos para la comunidad educativa, convertir el blog y las redes en una «ventana al mundo», referente de la discapacidad visual, abrirse a colaboraciones con profesionales de otros ámbitos y con todas aquellas personas que tengan relación con esta diversidad funcional y dar visibilidad a este colectivo.

La evolución de los seguidores en las redes sociales nos ha cogido por sorpresa. Desde luego, podemos decir que se están cumpliendo nuestros objetivos: «llegar al mayor número de personas posible», «compartir y dar a conocer materiales para que puedan ser usados por otros equipos educativos», etc. Todo ello nos ha motivado aún más, y estamos dispuestas a seguir nuestra labor con el objetivo de llegar a todos, porque, como escribió Antonio Machado: «En cuestiones de cultura y de saber, solo se pierde lo que se guarda; solo se gana lo que se da». 
María del Mar Montiel Campos. Maestra del Equipo Específico para la Atención al Alumnado con Discapacidad Visual de Málaga. Cuarteles, 8; 29002 Málaga (España). Correo electrónico: mamoca@once.es.

Esther Páez Romero. Maestra del Equipo Específico para la Atención al Alumnado con Discapacidad Visual de Málaga. Cuarteles, 8; 29002 Málaga (España).Correo electrónico: espr@once.es.

Amalia Vioque Fernández. Profesora del Equipo Específico para la Atención al Alumnado con Discapacidad Visual de Málaga. Cuarteles, 8; 29002 Málaga (España). Correo electrónico: amvf@once.es.

M. ${ }^{a}$ del Castillo Campanario Pérez. Maestra del Equipo Específico para la Atención al Alumnado con Discapacidad Visual de Málaga. Cuarteles, 8; 29002 Málaga (España). Correo electrónico: macper@once.es.

Mercedes Urbano Labajos. Maestra del Equipo Específico para la Atención al Alumnado con Discapacidad Visual de Málaga. Cuarteles, 8; 29002 Málaga (España).Correo electrónico: mul@ once.es. 